\title{
Multimodal Monitoring Strategy Is Decisive in Elective Middle Cerebral Artery Aneurysm Clipping: A Case Report
}

Dias, Sandra Fernandes ; Jehli, Elisabeth ; Regli, Luca ; Sarnthein, Johannes ; Germans, Menno R

\begin{abstract}
BackgroundbThe intraoperative use of neurophysiological monitoring (IONM) and indocyanine green video angiography (ICGVA) for aneurysm clipping have evolved during the last years. Both modalities are useful and safe by allowing greater rates of complete aneurysm occlusion with less intraoperative complications and postoperative neurologic deficits. We report a case of attempted aneurysm clipping in which the combined use of ICGVA and IONM was crucial for intraoperative decision-making. Case Descriptionb A 62-year-old woman was operated for an incidental 6-mm aneurysm at the origin of the right fronto-opercular branch. During aneurysm clipping, IONM amplitudes dropped drastically, despite patency of the parent artery and perforators in ICGVA. Several attempts for clipping were made with recurring drops in IONM amplitudes, which forced us to leave the aneurysm untreated. The patient had a postoperative left-sided hemiparesis that improved on follow-up. Thereafter, the aneurysm was treated with stent-assisted coiling. Conclusions The combination of IONM and ICGVA during aneurysm surgery allows for a better assessment of vascular integrity and patient's postoperative outcome than ICGVA alone. Simultaneous evaluation of vessel patency and integrity of the somatosensory and motor pathways illustrates the complementarity of testing different modalities for intraoperative decision-making and for maximizing safeness in aneurysm clipping.
\end{abstract}

DOI: https://doi.org/10.1016/j.wneu.2018.10.096

Posted at the Zurich Open Repository and Archive, University of Zurich

ZORA URL: https://doi.org/10.5167/uzh-158334

Journal Article

Accepted Version

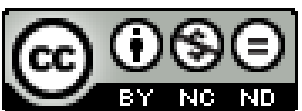

The following work is licensed under a Creative Commons: Attribution-NonCommercial-NoDerivatives 4.0 International (CC BY-NC-ND 4.0) License.

Originally published at:

Dias, Sandra Fernandes; Jehli, Elisabeth; Regli, Luca; Sarnthein, Johannes; Germans, Menno R (2019). Multimodal Monitoring Strategy Is Decisive in Elective Middle Cerebral Artery Aneurysm Clipping: A

Case Report. World Neurosurgery, 122:43-47.

DOI: https://doi.org/10.1016/j.wneu.2018.10.096 


\section{Accepted Manuscript}

Multimodal-monitoring strategy is decisive in elective middle cerebral artery aneurysm clipping: A case Report

Sandra Fernandes Dias, MD, Elisabeth Jehli, MSc, Luca Regli, MD, Johannes

Sarnthein, PhD, Menno R. Germans, MD, PhD

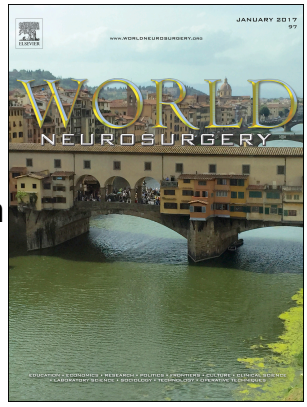

PII:

S1878-8750(18)32394-5

DOI:

https://doi.org/10.1016/j.wneu.2018.10.096

Reference: WNEU 10540

To appear in: World Neurosurgery

Received Date: 9 September 2018

Revised Date: 14 October 2018

Accepted Date: 16 October 2018

Please cite this article as: Dias SF, Jehli E, Regli L, Sarnthein J, Germans MR, Multimodal-monitoring strategy is decisive in elective middle cerebral artery aneurysm clipping: A case Report, World Neurosurgery (2018), doi: https://doi.org/10.1016/j.wneu.2018.10.096.

This is a PDF file of an unedited manuscript that has been accepted for publication. As a service to our customers we are providing this early version of the manuscript. The manuscript will undergo copyediting, typesetting, and review of the resulting proof before it is published in its final form. Please note that during the production process errors may be discovered which could affect the content, and all legal disclaimers that apply to the journal pertain. 
TITLE: Multimodal-monitoring strategy is decisive in elective middle cerebral artery aneurysm clipping: A case Report

\section{AUTHORS:}

Sandra Fernandes Dias ${ }^{1}, \mathrm{MD}$; Elisabeth Jehli ${ }^{1}, \mathrm{MSc}$; Luca Regli ${ }^{1}, \mathrm{MD}$; Johannes Sarnthein ${ }^{1,2}$, PhD; Menno R. Germans ${ }^{1}, \mathrm{MD}, \mathrm{PhD}$

${ }^{1}$ Department of neurosurgery, Clinical Neuroscience Center, University Hospital Zurich, University of Zurich, Zurich, Switzerland

${ }^{2}$ Zurich Neuroscience Center, ETH, Zurich, Switzerland

\section{Corresponding author:}

Sandra Fernandes Dias, MD

Department of Neurosurgery, University Hospital Zurich, University of Zurich, Zurich, Switzerland Frauenklinikstrasse 10 - $\mathrm{CH}-8091$ Zurich

Tel: +41-43-253 $8769 /+41-44-2551111 \quad$ Fax: +41-44-255 4387

E-mail: sandrafs.dias12@gmail.com

Abstract word count: 199

Text word count: 1516

Number of references: 18

Number of tables and/or figures: 4 


\section{ABSTRACT}

Background: The intraoperative use of neurophysiological monitoring (IONM) and indocyanine green video angiography (ICGVA) for aneurysm clipping have evolved during the last years. Both modalities are useful and safe by allowing higher rates of complete aneurysm occlusion with less intraoperative complications and postoperative neurological deficits. We report a case of attempted aneurysm clipping in which the combined use of ICGVA and IONM was crucial for intraoperative decision-making.

Case Description: A 62-y old female was operated for an incidental 6-mm aneurysm at the origin of the right fronto-opercular branch. During aneurysm clipping, IONM amplitudes dropped drastically, despite patency of the parent artery and perforators in ICGVA. Several attempts for clipping were made with recurring drops in IONM amplitudes, which forced us to leave the aneurysm untreated. The patient had a postoperative left-sided hemiparesis which improved on follow-up. Thereafter the aneurysm was treated with stent-assisted coiling.

Conclusions: The combination of IONM and ICGVA during aneurysm surgery allows for a better assessment of vascular integrity and patient's postoperative outcome than ICGVA alone. Simultaneous evaluation of vessel patency and integrity of the somatosensory and motor pathways, illustrates the complementarity of testing different modalities for intraoperative decision-making and for maximizing safeness in aneurysm clipping.

KeYwords: Aneurysm • Clipping • Indocyanine Green-Video Angiography • Intraoperative neurophysiological monitoring • Vascular neurosurgery 


\section{BACKGROUND}

Advances in microsurgical techniques, such as indocyanine green video angiography (ICGVA) and intraoperative neurophysiological monitoring (IONM), have made it possible to clip intracranial aneurysms with less risk of intraoperative complications and postoperative neurological deficits [1-4]. The use of IONM with both somatosensory (SSEP) and motor-evoked potentials (MEP) recording is increasingly being used in clipping of intracranial aneurysms, because the combination of both recordings has been shown to be highly predictive for postoperative neurological deficits $[5,6]$. Nevertheless, even with the standard use of ICGVA and the availability of IONM the risk for ischemia after clipping of unruptured aneurysms is reported as high as $6.7 \%$ [7].

Here we present a case of a patient with an attempted clipping of an incidental aneurysm of the fronto-opercular branch of the right middle cerebral artery (MCA), in which recurrent drops in IONM amplitudes occurred during repetitive clip placements, despite patency of the parent artery and lenticulostriate artery in ICGVA. The IONM results forced us not to clip the aneurysm.

\section{CASE DESCRIPTION}

A 62-year old female presented to our outpatient clinic with an unruptured 6-mm aneurysm of the right middle cerebral artery (figure 1). This incidental finding was diagnosed by CT-angiography, which was performed to assess the intracranial vasculature after ischemic stroke in the left middle cerebral artery territory. The broad based non-calcified saccular aneurysm originated from the fronto-opercular branch of the M1 and was oriented posterior-lateral. A lenticulostriate artery was seen, arising from the proximal part of the fronto-opercular branch. This right-handed patient suffered from arterial hypertension, did not smoke and had a negative family history for intracranial aneurysms. Clinical examination revealed no neurological deficits. After discussion in our multidisciplinary team, and considering the aneurysm's characteristics (broad-based, presence of a proximal lenticulostriate artery) as well as patient's preference, it was decided to treat the aneurysm by microsurgical clipping, instead of coiling. 
During surgery, we performed continuous IONM using ISIS system (inomed GmbH, Emmendingen, Germany). For SSEP monitoring, monophasic square-wave electrical pulses (repetition frequency $4.7 \mathrm{~Hz}$ ) were delivered at the median nerve (intensity 20 $\mathrm{mA})$ and tibial nerve $(40 \mathrm{~mA})$. The SSEP responses were obtained through corkscrew electrodes placed at electrode sites C3'/C4' versus Cz/Fz and filtered (20$300 \mathrm{~Hz}$ ) before display.

For MEP monitoring, the current for transcranial electrical stimulation (TES) was delivered through 2 corkscrew electrodes placed at electrode sites $\mathrm{C} 1$ and $\mathrm{C} 2$ on the scalp. Constant current stimulation $(120 \mathrm{~mA})$ was applied in trains of 5-7 pulses (width $0.5 \mathrm{msec}$ ) with an stimulus interval of $4 \mathrm{msec}$. MEP responses (filtered 100$3000 \mathrm{~Hz}$ ) were obtained bilaterally from the thenar and flexor hallucis brevis muscles. The surgical team was notified whenever the MEP and/or SSEP amplitude dropped below $50 \%$ or MEP intensity threshold had to be elevated more than $20 \mathrm{~mA}$ compared to baseline.

The patient was positioned in supine position with the head slightly rotated to the left in a Mayfield head-holder. A right-sided latero-supraorbital craniotomy was performed according to institutional practice. The Sylvian fissure was dissected to expose the M1 branch and the smaller fronto-opercular branch with the lateral lenticulostriate artery. A first drop of MEP amplitude (figure 2) was attributed to deeper anaesthesia and MEP stimulation intensity was increased to $130 \mathrm{~mA}$. During aneurysm preparation, the lateral lenticulostriate artery arising from the proximal part of the superior trunk was identified. A permanent Yasargil clip (FT746T) was applied perpendicular to aneurysm's neck, to preserve flow into perforators.

After aneurysm clipping with satisfactory anatomical result, ICGVA was performed with a standard dose of $0.3 \mathrm{mg} / \mathrm{kg}$ of $25 \mathrm{mg}$ ICG-powder dissolved in $5 \mathrm{ml}$ of water, injected into a central vein [8]. ICGVA showed patency of the parent artery, distal M1 trunk, fronto-opercular branch, and the lateral lenticulostriate artery (figure 3).

Approximately one minute later, a significant drop on MEP's response and SSEP's amplitudes was seen. This prompted us to remove the clip, after which the MEP and SSEP amplitudes normalized rapidly. Despite several attempts for clipping with different types of clips and clipping techniques, IONM amplitudes kept dropping drastically. Amplitudes always recovered after removal of the clip. At the end of surgery, MEP stimulation intensity $(+20 \mathrm{~mA})$ and the number of pulses $(+2)$ had to be 
elevated, indicating potential postoperative neurological deficits. Due to the repetitive decreases in MEP and SSEP amplitudes we decided to leave the aneurysm untreated.

Postoperatively, the patient had a left hemiplegia, recovering into a severe paresis few hours later. Control MRI showed an infarct on the right lenticular nucleus and posterior limb of the internal capsule (figure 4A). The MCA, fronto-opercular branch and lateral lenticulostriate artery were still patent (figure 4B \& $C$ ). The patient was discharged into a rehabilitation centre. Follow-up at eight months showed significant recovery with only slight disturbances of the fine motor skills of the left hand and the patient was able to walk approximately 15 minutes without limitations (modified Rankin scale score 2). Because at 8-months follow-up the aneurysm had increased in size we re-discussed the case at the multidisciplinary board. The decision was to treat the aneurysm by stent-assisted coiling. This procedure was successfully performed two months later.

\section{DISCUSSION}

\section{$\underline{\text { Value of vessel patency assessment - ICGVA }}$}

Microscope integrated near infrared ICGVA is a simple, fast, and non-invasive technique that allows intraoperative spatial and temporal resolution of arterial and venous phases in small and big vessels [2, 9-13]. Besides being a safe technique, ICGVA has many utilities on the management of intracranial aneurysms by allowing to assess aneurysm anatomy, patency of distal branches and perforating vessels $(<0.5-\mathrm{mm})$, flow-direction and real-time confirmation of aneurysm's exclusion [12, 14]. The advantages of this technique in vascular neurosurgery encompass also the correct identification of donor and recipient vessels in case of revascularization procedures. Although no complications have been so far reported with the use of ICGVA, some limitations as the time-consuming character with possibility of slightly longer temporary occlusion time to perform ICGVA - with increased risk of ischemic complications - should be taken into account [11, 15].

\section{Considerations for intraoperative monitoring}

The increased experience with IONM has shown that changes in sensory and motor function have a predictive capacity for postoperative neurological status of the 
patient. Particularly the use of SSEPs in clipping unruptured aneurysms is improving safeness and recent studies showed a high sensitivity [6, 16]. A cohort study, which analysed the use of IONM during treatment of 691 intracranial aneurysms, showed a higher sensitivity of SSEP changes in predicting postoperative stroke in the surgical treatment of unruptured aneurysms, in comparison to the ruptured aneurysms [17]. Regarding the use of IONM on the particular case of aneurysms of the MCA, a recent multivariate analysis showed that the use of MEPs is an independent prognostic factor for motor outcome in long-term follow-up, by allowing a reliable assessment of ischemia at the level of the cortico-spinal tract [18].

\section{Multimodal strategy}

We presented a case of a patient with an incidental aneurysm of the right MCA at the fronto-opercular branch in which the use of intraoperative multimodal assessment lead to the decision not to clip the aneurysm, in order to preserve patient's neurological integrity. Despite an anatomically satisfactory result and confirmation of the filling of the distal M1 trunk, fronto-opercular branch and lenticulostriate artery by ICGVA, the aneurysm was not clipped due to significant changes in IONM.

Della Puppa et al [4] performed the first analysis of multimodal strategy in aneurysm clipping. They showed the relevance of concomitant use of IONM, ICGVA and flowmetry in achieving a maximal rate of aneurysm occlusion, with the lowest morbidity. Their data showed that distal aneurysms (MCA) were more prone to have significant flow decrease on flowmeter; while aneurysms of the anterior circulation were at higher risk for MEP impairment $(P<0.05)$. The MCA-aneurysm from our patient would have be dichotomized as belonging to the lower (distal group) risk of perforating arteries injury. On our opinion, the possible origin of the postoperative infarct could have been related to the repetitive ischemic insults induced by the several attempts to clip the aneurysm. The clip removal allowed a reperfusion of the posterior limb of the internal capsule and corona radiata with recovery of the MEPs. This points out the relevance of our case report regarding the complementarity of information provided by the simultaneous use of IONM and ICGVA and the challenge in intraoperative decision making.

Although ICGVA and microsurgical inspection showed that anatomically the vessels were patent, changes in IONM recordings indicated vascular compromise. In order to prevent severe postoperative permanent deficits we decided to leave the aneurysm 
untreated. Ten months later, after radiological evidence of aneurysm growth, the decision for endovascular treatment of the aneurysm was made. After selective catheterisation of the inferior trunk, and placement of a $4 \times 20-\mathrm{mm}$ stent the aneurysms was successfully coiled, even though a small perfusion rest was still observed.

\section{CONCLUSIONS}

The use of concomitant IONM and ICGVA during aneurysm surgery allows better prediction of vascular integrity and possibly improves outcome than ICGVA alone. The simultaneous evaluation of vessel patency and integrity of the somatosensory and motor pathways, illustrates the complementarity of testing different modalities for intraoperative decision-making and for maximizing safeness in aneurysm clipping.

\section{FUNDING}

No funding was received for this research.

\section{ETHICAL APPROAVAL}

This study was approved by the institutional ethics review board (Kantonale Ethikkommission KEK-ZH-2012-0212). All data presented was anonymized. 


\section{REFERENCES}

1. Grasso, G., A. Landi, and C. Alafaci, Multimodal Intraoperative Neuromonitoring in Aneurysm Surgery. World Neurosurg, 2017. 101: p. 763-765.

2. Raabe, A., et al., Near-infrared indocyanine green video angiography: a new method for intraoperative assessment of vascular flow. Neurosurgery, 2003. 52(1): p. 132-9; discussion 139.

3. Schramm, J., J. Zentner, and U. Pechstein, Intraoperative SEP monitoring in aneurysm surgery. Neurol Res, 1994. 16(1): p. 20-2.

4. Della Puppa, A., et al., Microsurgical Clipping of Intracranial Aneurysms Assisted by Neurophysiological Monitoring, Microvascular Flow Probe, and ICG-VA: Outcomes and Intraoperative Data on a Multimodal Strategy. World Neurosurg, 2018. 113: p. e336-e344.

5. Spina, A. and P. Mortini, Intraoperative Neuromonitoring Strategies for Temporary Clipping in Aneurysm Surgery. World Neurosurg, 2017. 101: p. 773-775.

6. Staarmann, B., et al., Sensitivity and Specificity of Intraoperative Neuromonitoring for Identifying Safety and Duration of Temporary Aneurysm Clipping Based on Vascular Territory, a Multimodal Strategy. World Neurosurg, 2017. 100: p. 522-530.

7. Alshekhlee, A., et al., Hospital mortality and complications of electively clipped or coiled unruptured intracranial aneurysm. Stroke, 2010. 41(7): p. 1471-6.

8. Esposito, G., et al., Indocyanine green videoangiography for the identification of superficial temporal artery branches in EC-IC bypass surgery. Acta Neurochir (Wien), 2016. 158(3): $p$. 565-70.

9. Dashti, R., et al., Microscope-integrated near-infrared indocyanine green videoangiography during surgery of intracranial aneurysms: the Helsinki experience. Surg Neurol, 2009. 71(5): p. 543-50; discussion 550.

10. de Oliveira, J.G., et al., Assessment of flow in perforating arteries during intracranial aneurysm surgery using intraoperative near-infrared indocyanine green videoangiography.

Neurosurgery, 2007. 61(3 Suppl): p. 63-72; discussion 72-3.

11. Esposito, G., et al., Selective-targeted extra-intracranial bypass surgery in complex middle cerebral artery aneurysms: correctly identifying the recipient artery using indocyanine green videoangiography. Neurosurgery, 2012. 71(2 Suppl Operative): p. ons274-84; discussion ons284-5.

12. Scerrati, A., et al., Indocyanine green video-angiography in neurosurgery: a glance beyond vascular applications. Clin Neurol Neurosurg, 2014. 124: p. 106-13.

13. Raabe, A., et al., Prospective evaluation of surgical microscope-integrated intraoperative nearinfrared indocyanine green videoangiography during aneurysm surgery. J Neurosurg, 2005. 103(6): p. 982-9.

14. Esposito, G. and L. Regli, Selective Targeted Cerebral Revascularization via Microscope Integrated Indocyanine Green Videoangiography Technology. Acta Neurochir Suppl, 2014. 119: p. 59-64.

15. Roessler, K., et al., Essentials in intraoperative indocyanine green videoangiography assessment for intracranial aneurysm surgery: conclusions from 295 consecutively clipped aneurysms and review of the literature. Neurosurg Focus, 2014. 36(2): p. E7.

16. Kashkoush, A.I., et al., Somatosensory Evoked Potentials During Temporary Arterial Occlusion for Intracranial Aneurysm Surgery: Predictive Value for Perioperative Stroke. World Neurosurg, 2017. 104: p. 442-451.

17. Wicks, R.T., et al., Impact of changes in intraoperative somatosensory evoked potentials on stroke rates after clipping of intracranial aneurysms. Neurosurgery, 2012. 70(5): p. 1114-24; discussion 1124.

18. Yue, Q., et al., Motor evoked potential monitoring during surgery of middle cerebral artery aneurysms: a cohort study. World Neurosurg, 2014. 82(6): p. 1091-9. 


\section{FIGURE/CAPTIONS LEGENDS}

Figure 1. Pre-operative imaging of the aneurysm. A. Preoperative CTA showing the right-sided middle cerebral artery aneurysm arising at the fronto-opercular branch. B. 3D-Reconstruction of the aneurysm with a small lenticulostriatal artery arising from its proximal part.

Figure 2. IONM during the course of surgery. MEP and SSEP amplitudes of the left side of the body dropped after each attempt to clip the aneurysm and recovered after removal of the clip. $\propto$ Clip application.

Figure 3. Intraoperative clipping of the aneurysm. A. Anatomically satisfactory result of aneurysm clipping $\mathbf{B}$. Left panel - microscopic field of view with identification of the parent artery (origin of the fronto-opercular branch), both proximal and distal trunks of the middle cerebral artery and the lenticulostriate artery at the base of the fronto-opercular branch. b. Right panel - Intraoperative ICG-VA showing patency of all vessels after clip placement.

$\propto$ Permanent clip; \# Right proximal middle cerebral artery; \#M1d: distal middle cerebral artery trunk; \#FOP: fronto-opercular branch of the MCA; * lenticulostriate artery

Figure 4. Post-operative imaging. A. Postoperative MRI revealing the infarct zone at the posterior part of the right lenticular nucleus, posterior limb of the internal capsule and corona radiata B \& C. MRI/MRA showing patency of the untreated aneurysm and perforator coming from its basis (see arrow). 


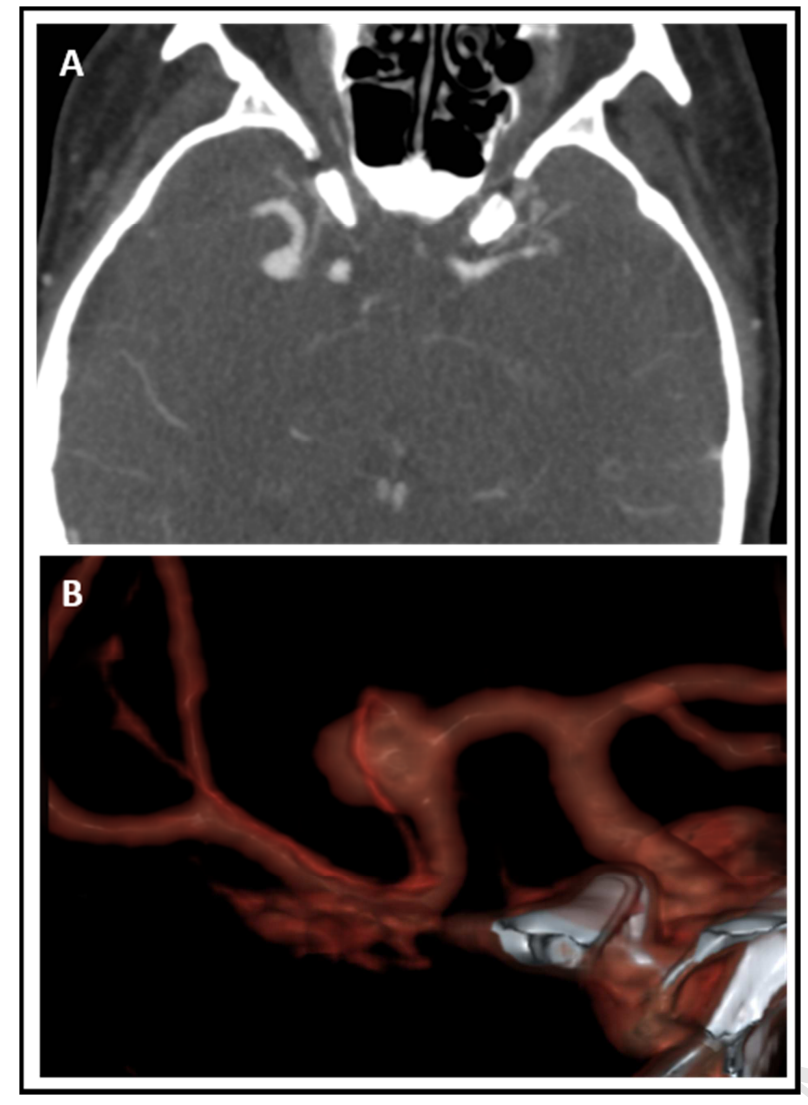




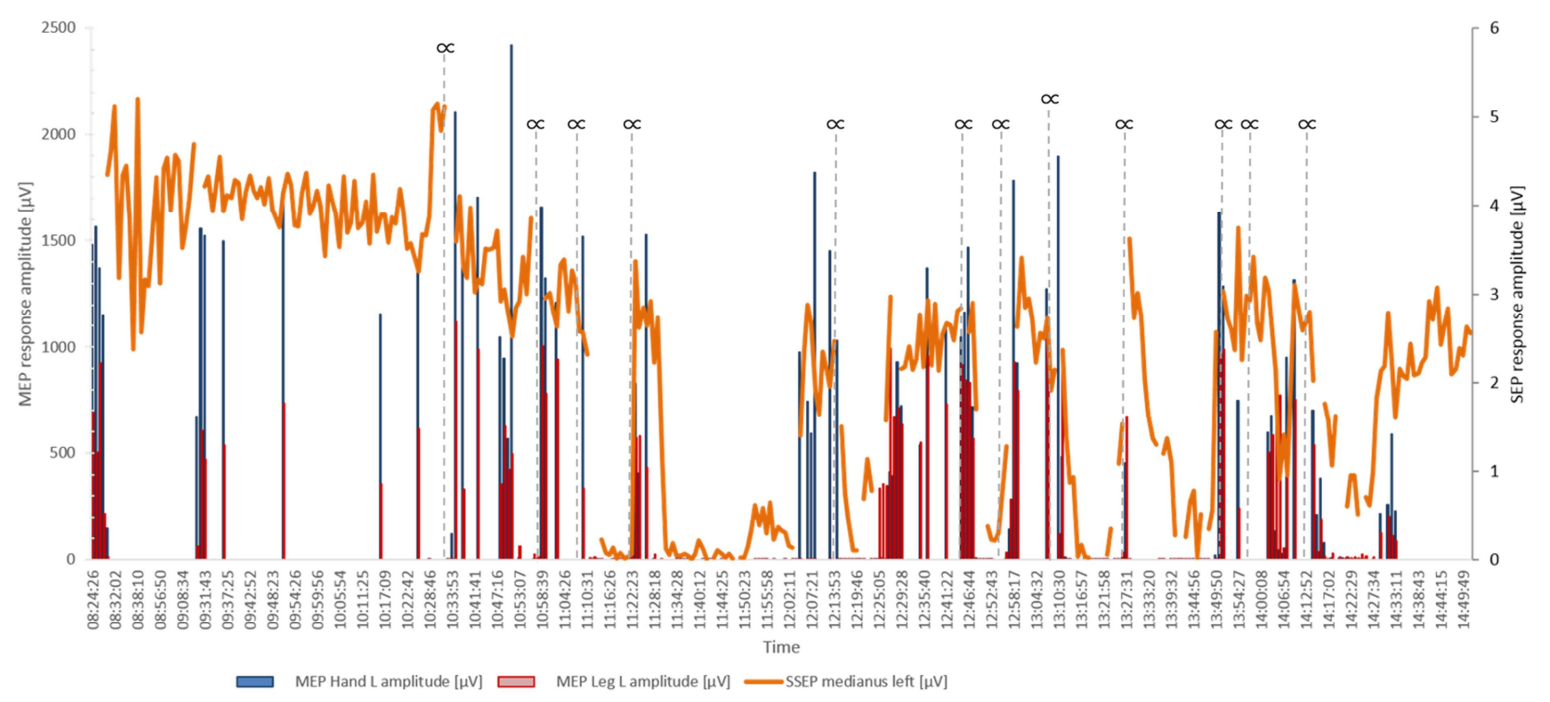




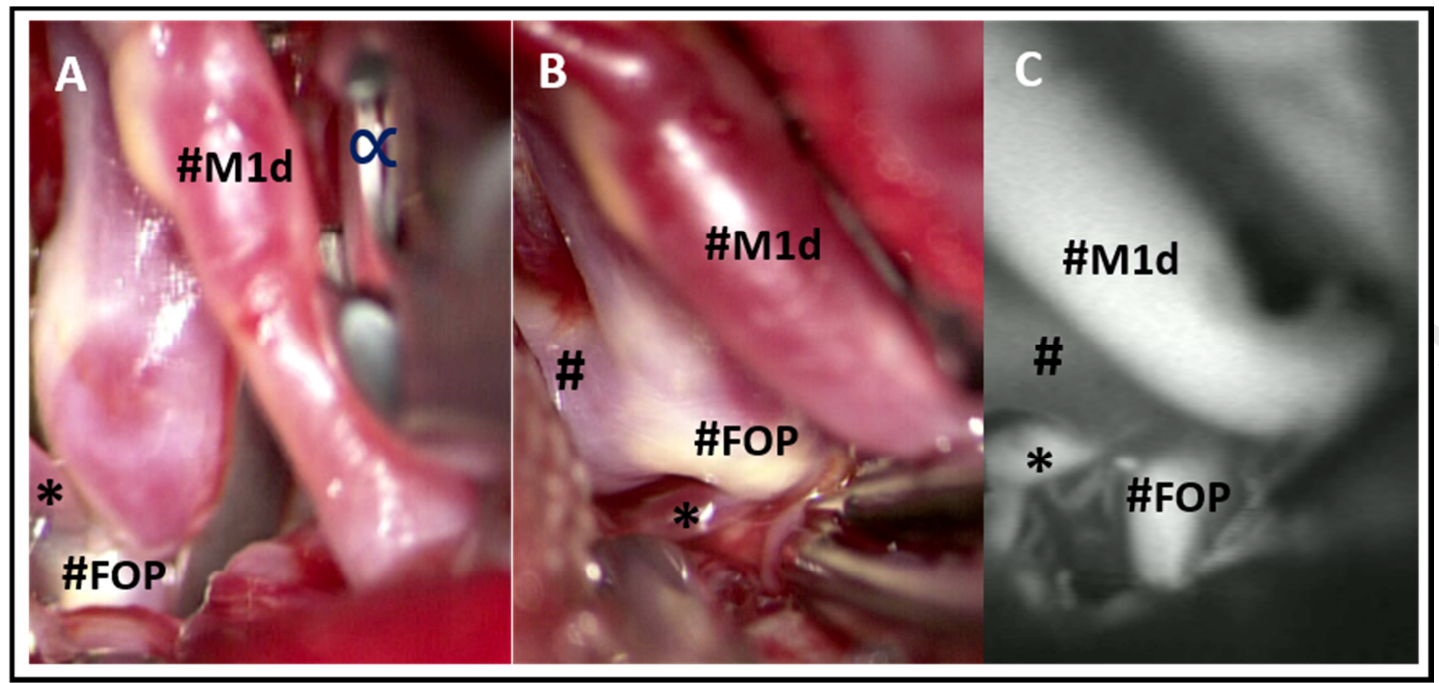




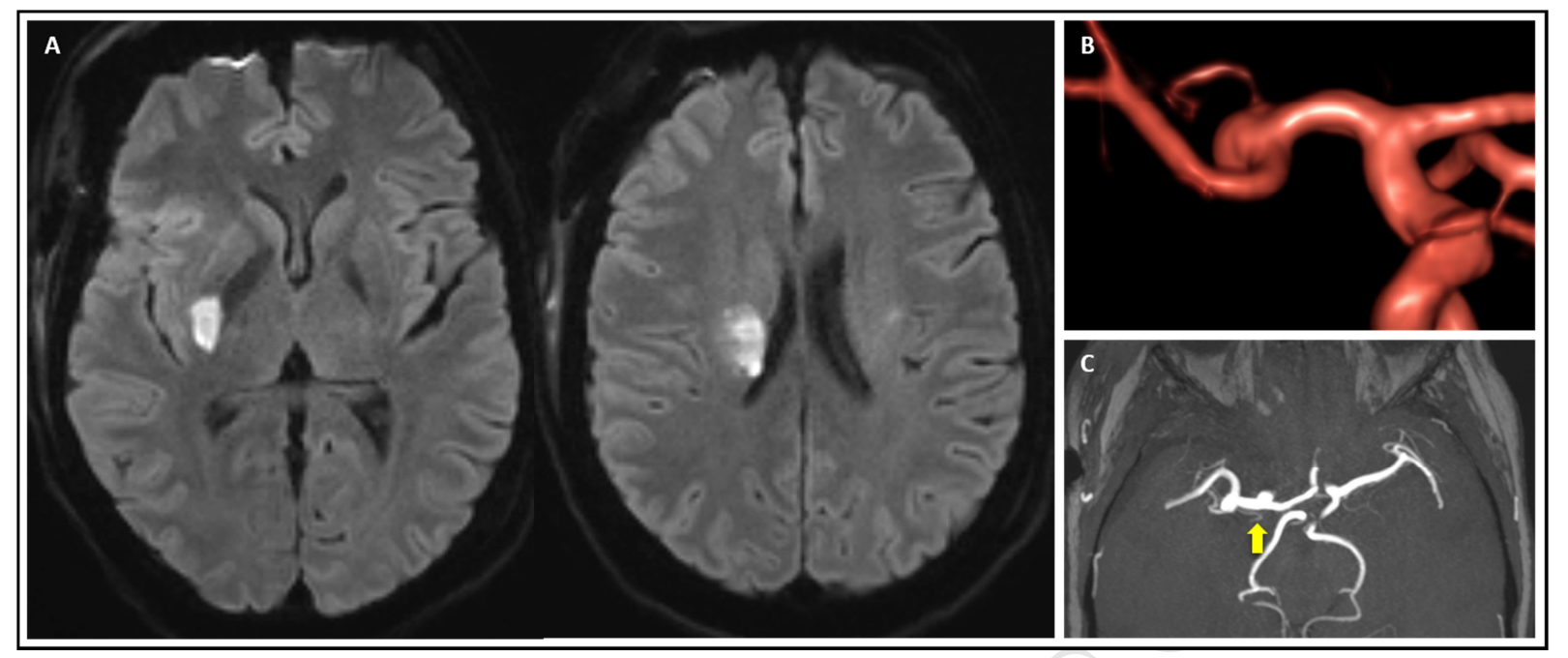




\section{Abbreviations}

- MCA: middle cerebral artery

- MRI: magnetic resonance imaging

- CTA: computer tomography angiography

- IONM: intraoperative neurophysiological monitoring

- MEP: motor evoked potential

- SSEP: somatosensory evoked potential

- ICGVA: indocyanine green video-angiography

- mRS: modified ranking scale 